\title{
FORMATION OF ATOMIC CLUSTERS THROUGH THE LASER ABLATION OF REFRACTORY MATERIALS IN A SUPERSONIC MOLECULAR BEAM SOURCE
}

\author{
R. E. Haufler, A. A. Puretzky ${ }^{\circ}$, and R. N. Compton \\ Oak Ridge National Laboratory \\ Post Office Box 2008, Oak Ridge, TN 37831-6125
}

\begin{abstract}
Concepts which guide the design of atomic cluster supersonic beam sources have been developed. These ideas are founded on the knowledge of laser ablation dynamics and are structured in order to take advantage of certain features of the ablation event. Some of the drawbacks of previous cluster source designs become apparent when the sequence of events following laser ablation are clarified. Key features of the new cluster source design include control of the cluster size distribution, uniform performance with a variety of solid materials and elements, high beam intensity, and significant removal of internal energy during the supersonic expansion.
\end{abstract}

\section{INTRODUCTION}

The method of generating usable beams of cold atomic clusters via laser ablation of materials in a supersonic molecular beam source was first introduced over ten years ago by the Smalley Group at Rice University ${ }^{1}$. While the clusters produced in these sources have attracted considerable theoretical and experimental attention, the literature is surprisingly sparse where the production of such species is concerned. New experimental approaches ${ }^{2}$ have probed ablation phenomenon and have provided significant insight furthering the understanding of the processes occurring in the supersonic cluster beam sources. Armed with increasing knowledge of laser induced plasmas combined with the well established field of supersonic molecular beam gas dynamics, guiding concepts in the design of these sources are beginning to emerge.

\section{MECHANISM}

The processes following the laser ablation event involve complex chemical condensation reactions occurring in a laser generated plume interacting with the surrounding buffer gas. This complex chemistry is followed by supersonic expansion of the material into vacuum. These clusters are covalent or ionic and are stable at elevated temperatures by contrast with weakly bound van der Waals clusters. The condensation and cluster formation occurs in the nozzle volume prior to the

- also of Institute of Spectroscopy, Russian Academy of Sciences, 142092 Troitsk, Moscow, Russia

\section{MASTER}

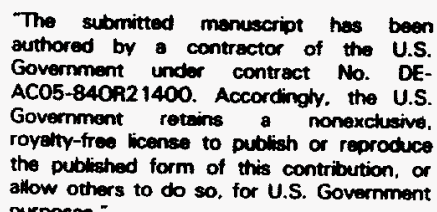




\section{DISCLAIMER}

This report was prepared as an account of work sponsored by an agency of the United States Government. Neither the United States Government nor any agency thereof, nor any of their employees, make any warranty, express or implied, or assumes any legal liability or responsibility for the accuracy, completeness, or usefulness of any information, apparatus, product, or process disclosed, or represents that its use would not infringe privately owned rights. Reference herein to any specific commercial product, process, or service by trade name, trademark, manufacturer, or otherwise does not necessarily constitute or imply its endorsement, recommendation, or favoring by the United States Government or any agency thereof. The views and opinions of authors expressed herein do not necessarily state or reflect those of the United States Government or any agency thereof. 


\section{DISCLAIMER}

Portions of this document may be illegible in electronic image products. Images are produced from the best available original document. 
expansion. The supersonic expansion has the purpose of cooling the clusters and it is assumed that little or no further cluster growth occurs due to the expansion.

The dominant problem encountered in these sources is the shock wave confinement of the plume. This has the undesired effect of promoting wholesale condensation of the ablated material and a delay in the mixing of the plume species with the carrier gas. Mixing with the carrier gas leads to better cooling in the supersonic expansion and more control of the cluster size distribution by lowering the rate of condensation through dilution effects. These concepts are also pertinent when this approach is used to produce seeded beams of molecules and bio-molecules using laser desorption (LD) and matrix assisted laser desorption (MALD).

A solution becomes apparent when considering the ablation of the material of interest in vacuum. In the absence of a surrounding buffer gas the shock wave does not form allowing the plume to expand unimpeded and to rapidly dissipate. Swift pressurization of the nozzle volume at the point where the pressure of the plume is near the desired nozzle pressure when diffusion becomes the dominant transport mechanism leads to better mixing.

\section{BACKGROUND}

A key parameter in affecting the optimum geometry of the cluster source is the time the material spends in the source volume prior to supersonic expansion into vacuum. The pumping speed through the nozzle orifice can be determined by

$$
s=A v \text {, }
$$

where $s=$ the pumping speed, $A=$ orifice cross-sectional area, and $v=$ the speed of sound for the nozzle gas. The speed of sound is determined by

$$
v=\sqrt{\gamma \frac{k T}{m}}
$$

where $\gamma=$ the heat capacity ratio of the buffer gas, $k=$ Boltzmann's constant, $T=$ the stagnation temperature of the nozzle gas, and $m=$ mass of the gas. The temperature is not necessarily that of the nozzle apparatus due to the additional energy imparted by the laser. The residence time, $t r$, that the ablated material spends in the nozzle source of volume $V$ before the supersonic expansion can be estimated by equation 3 :

$$
t_{r}=\frac{V}{S}
$$

Thus for a cylindrically symmetric cluster source of $2 \mathrm{~mm}$ diameter, $8.275 \mathrm{~mm}$ length and an exit orifice diameter of $1.5 \mathrm{~mm}$, the time that the ablated material has to form 


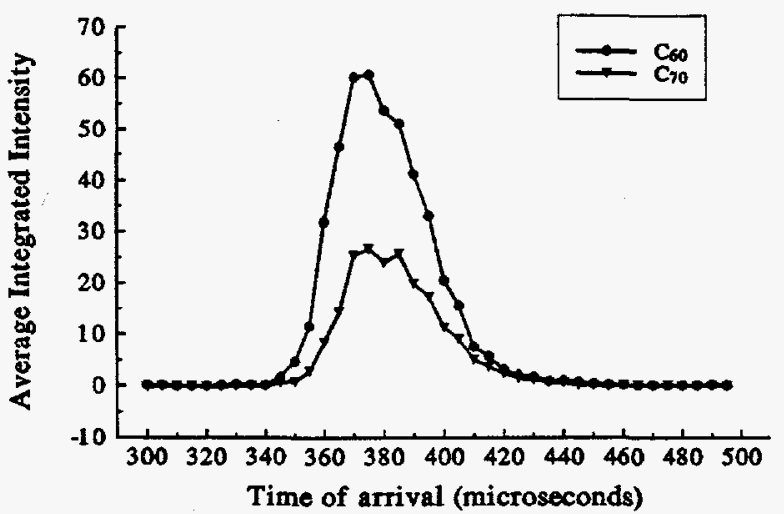

Figure 1. TOA profile for $\mathrm{C}_{60}$ and $\mathrm{C}_{70}$ from the nozzle source to the mass spectrometer. The value of tr for the nozzle source in this case was 35 microseconds and the distance traveled was 1 meter. clusters is roughly 14 microseconds. The residence time of the clusters in the nozzle is revealed by the time-ofarrival profile of the clusters at some point on the molecular beam axis. One such TOA profile for laser desorbed $\mathrm{C}_{60}$ and $\mathrm{C}_{70}$, measured using a mass spectrometer is shown in figure 1. Generally, longer residence time $t r$ promotes more extensive condensation. Equation 4 illustrates the relationship between supersonic velocity, Uss, and the Mach number, $M^{3}$

$$
u_{s s}{ }^{2}=\frac{2 k T}{m}\left(1+\frac{\gamma-1}{2} M^{2}\right)^{-1} \frac{\not M^{2}}{2} .
$$

For a monoatomic gas, this equation simplifies to equation 5 in the limit of infinite Mach number:

$$
v_{s s}=\sqrt{\frac{5 k T_{o}}{m}},
$$

The dependence of the supersonic velocity on the Mach number is presented in figure

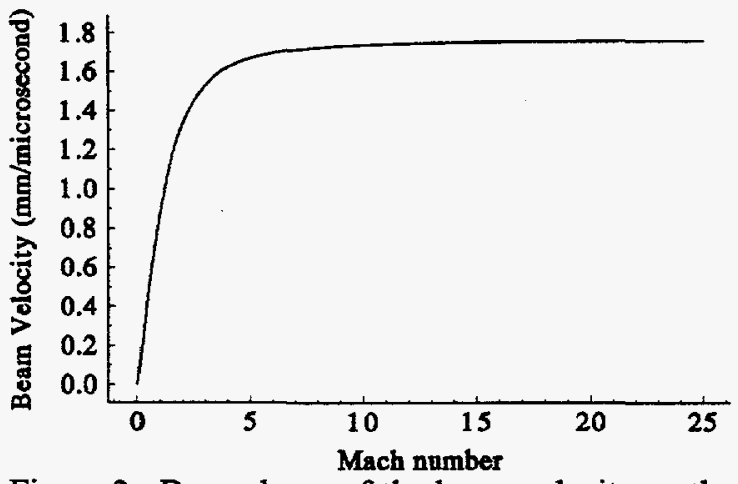

Figure 2. Dependence of the beam velocity on the terminal Mach number for $300 \mathrm{~K}$ helium.
2. Measurement of the supersonic velocity provides a means of estimating the stagnation temperature, $T o$. For the case in figure 1 , this temperature was $660 \mathrm{~K}$. This is not the final temperature of the clusters but rather it is the temperature prior to the supersonic expansion. Free jet expansion further cools the clusters. This information provides a relative indicator when optimizing other parameters.

An alternative method for detection of neutral species from the pulsed supersonic cluster beam source is through the use of a special pyroelectric detector. ${ }^{4}$ Figure 3 shows oscilloscope traces obtained using this detector for the ablation of graphite in the nozzle using $\mathrm{XeCl}$ excimer laser radiation $(\lambda=308 \mathrm{~nm}$, pulse width $=25$ nanoseconds, fluence $=30$ millijoules $/ \mathrm{mm}^{2}$ ). The top trace was obtained for the carrier gas only. The middle and bottom traces were obtained when the laser pulse 
Figure 3. Oscilloscope traces obtained from pyroelectric detector placed on molecular beam axis (see text).

was synchronized and delayed 84 microseconds (figure 3 , middle trace) and 100 microseconds (figure 3 , bottom trace) relative to the opening of the pulsed gas valve. Two interesting effects are observed: (1) the single pulse becomes two (figure 3 , middle trace) the first has an earlier arrival time and the second which appears to be slightly slower and (2) the pulse narrows as the gas pulse/laser pulse delay is slightly increased (figure 3, bottom trace). An explanation follows. The ablation event heats approximately $1 / 3$ of the gas in a single pulse in the volume of the nozzle and also adds material to the gas through the ablation of the graphite target. Assuming equilibrium and in accordance with equation (5), the mixture of gas, due to the higher temperature, will have an increased velocity. Thus the peak at time preceding the carrier gas peak is observed. The carrier gas peak appears to be slightly delayed. This may be understood when considering a fraction of the material originally in the pulse has become part of the new peak. Thus the front edge of the original peak has lost some intensity. The narrowing of this feature observed when the delay was increased to 100 microseconds is attributed to the increased cooling of the ablated material due to the higher density of the carrier gas at later delays.

The choice of a valve for optimum cluster formation and cooling is critical. The primary prerequisite is the rapid delivery of a large volume of gas. Our choice was the commercially availables "hairpin" type pulsed supersonic nozzle valve. ${ }^{6}$ The action of the valve is contained in two copper beryllium leaf springs which have a fundamental frequency of $4 \mathrm{kHz}$. A high current (5000 amps, 30 microseconds) is pulsed through

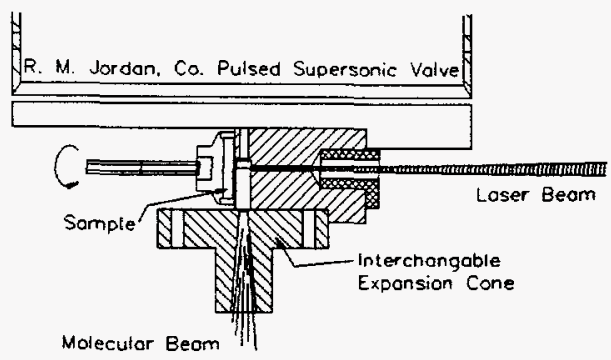

Figure 4. Side vaporization source using a single valve and disk samples. the springs in opposing directions. The magnetic field generated by the pulsed current forces the spring apart thus breaking the seal. The moving parts in this case are of small mass and can move very fast. The pulse width can be varied from 50 the 125 microseconds. The exit orifice diameter of the valve in this case was $0.75 \mathrm{~mm}$. The commercial valve is cylindrical in shape, $90 \mathrm{~mm}$ diameter, $200 \mathrm{~mm}$ length. This shape proved awkwardly large 
and a smaller, rectangular valve design was developed based on the same principles. MECHANICAL CONFIGURATIONS

Two examples of the many possible configurations of the supersonic cluster beam source are depicted in figures 4 and 5 . Figure 4 shows a side vaporization source using a single pulsed valve. This particular configuration uses samples in disk forms. In figure 5, examples of two valve schemes are shown. These sources have the vaporization laser co-axial with the molecular beam. The advantage of this is that there is no hole in the side for the ablation laser. Clusters are lost through the side

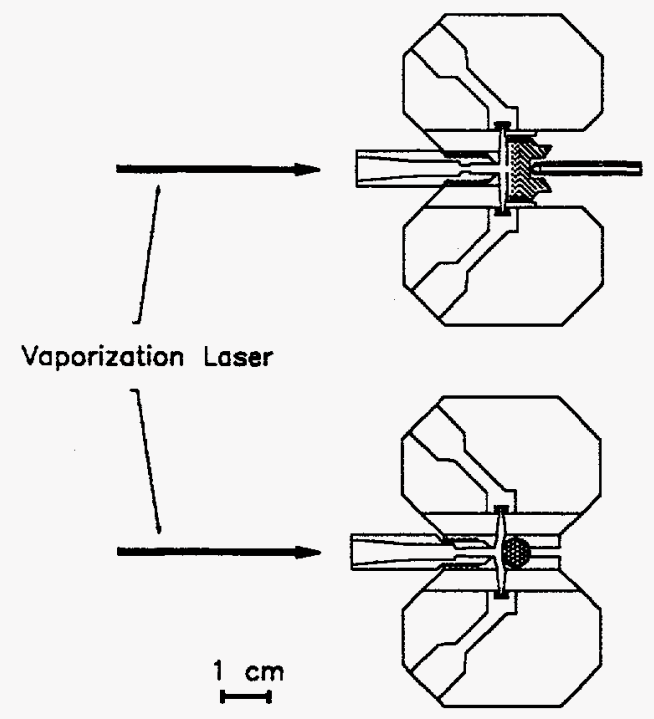

Figure 5. Two valve schemes using disk samples (top) and rod samples (bottom) which take advantage of on-axis vaporization holes thus there is a great advantage to the on-axis configuration. Additionally, the velocity of the expanding plume has the correct direction. The two valves provide greater source pressure for additional cooling. The advantages of samples in flat or disk forms over round rod-shaped forms is utility. Many materials are available in wafers or foils and much less material is needed. The disadvantages are that the sample motion is more complicated (although this can usually be overcome). An additional advantage of the disk format is the ability to perform laser desorption and matrix assisted laser desorption on sample deposited disks. It is also possible to deposit the sample onto rods. One disadvantage of the rod is that the sample can be easily scraped off.

\section{RESULTS}

The parameters affecting optimum cluster source design is best described through a few examples. Figure 6 shows the dependence of the cluster size distribution on the time delay between the pressurization of the nozzle volume and the ablation event. The higher nozzle pressure resulting from the increased in delay led to more extensive cluster growth.

Figure 7 shows another example for the laser ablation of carbon. In this case, early time delays for which the ablation occurs when the nozzle pressure is low, the carbon clusters are produced as positive ions. Additionally, significant amounts of oddnumbered clusters are detected in the fullerene size range. The early delay abundance distribution favors the smaller clusters as in the case of niobium. This suggests that the plume species growth reactions are quenched allowing detection of typically unstable and unobserved clusters. Neutral clusters are produced when the source is pressurized 35 microseconds prior to the ablation event (see fig. 7). In this case, the 


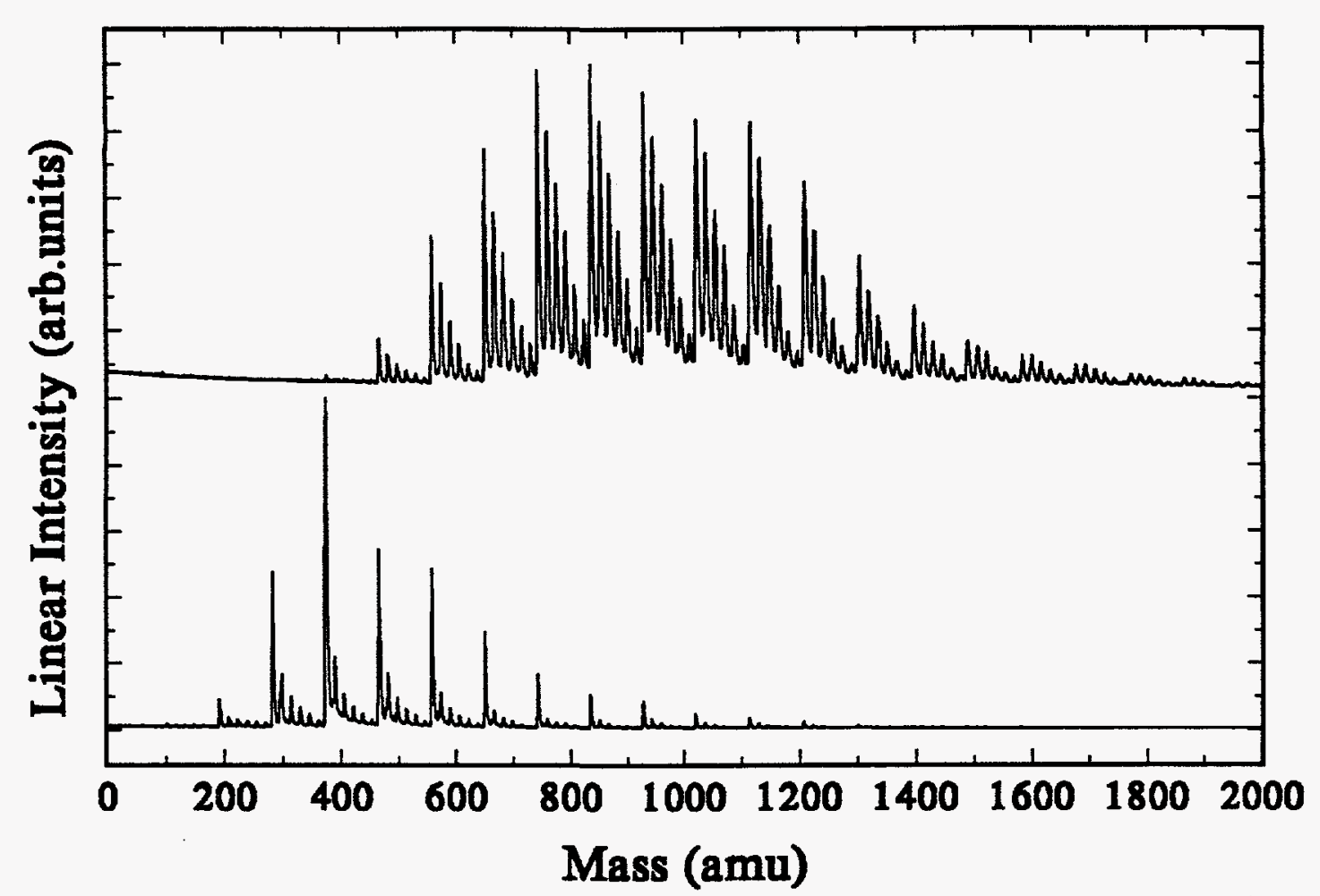

Figure 6. Reflectron time-of-flight mass spectrum of niobium clusters with $193 \mathrm{~nm}$ excimer laser ionization. The top distribution was obtained when pressurization preceded ablation by 5 microseconds. Zero delay conditions produced the bottom spectrum. Oxides of the niobium clusters are also detected.

familiar fullerene cluster size distribution is observed. Interestingly, the most abundant small cluster present was $\mathrm{C}_{28} . \quad \mathrm{C}_{28}$ is not usually observed as a product from the fragmentation of fullerenes. ${ }^{7}$ Therefore, this cluster must be produced through growth processes. There exists a good tetrahedral structure for this cluster which is predicted to be quite stable if properly passivated. This structure has four sites where three pentagons are joined by a single carbon atom. The atom at this site has $\mathrm{sp}^{3}$ geometry thus will be a good site for attack behaving as a dangling bond or unsatisfied valence. Structures where the $\mathrm{C}_{28}$ cluster has grown about single tetravalent metal atoms, uranium, zirconium, and titanium, have been observed and produced in milligram quantities. ${ }^{8}$ Note that there are the ions $\mathrm{C}_{28} \cdot(\mathrm{Ar})_{n}{ }^{+}$and $\mathrm{C}_{32} \cdot(\mathrm{Ar})_{n}{ }^{+}$, where $\mathrm{n}=1,2$; present in the mass spectrum. These species were demonstrated to be van der Waals species through laser induced photofragmentation. The bond energy of the carbon cluster/noble gas atom is very small, less than $1000 \mathrm{~cm}^{-1}$. This is a remarkable demonstration of the ability of this technique to cool ablated species. The temperatures generated in the ablation of graphite may approach $10,000 \mathrm{~K}$. Thus this source, with its ability to allow the plume to mix with the carrier gas and the subsequent expansion has cooled the ablated material from $10,000 \mathrm{~K}$ to $\sim 50 \mathrm{~K}$ in less than 100 microseconds.

Visible resonant two-photon ionization spectroscopy of laser desorbed $\mathrm{C}_{60}$ and $\mathrm{C}_{70}$ measured using a two valve on-axis configuration revealed very sharp transitions 


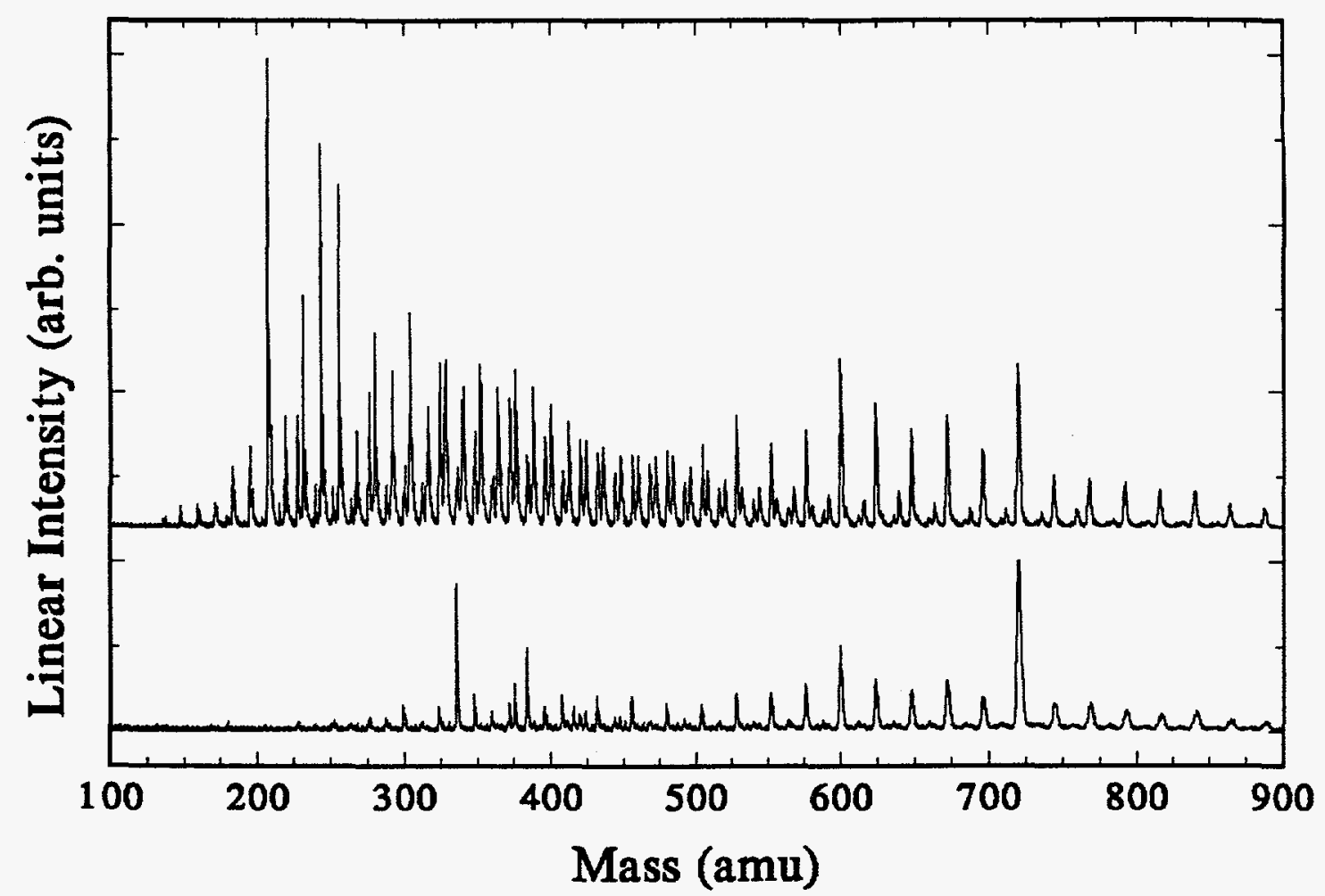

Figure 7. Reflectron time-of-flight mass spectrum of carbon clusters produced in the laser ablation of graphite. The top distribution was obtained with zero delay between pressurization and ablation. In this case the clusters produced were positively charged The bottom spectrum was obtained when pressurization preceded ablation by 35 microseconds. In this case only neutrals were produced which were $157 \mathrm{~nm}$ excimer laser ionized. The buffer gas was helium with $1 \%$ argon and some $\mathrm{C}_{\mathrm{n}} \cdot \mathrm{Ar}_{\mathrm{x}}$ clusters are detected.

which were narrower than $0.2 \mathrm{~cm}^{-1}$ in the range 5000 to 6000 Angstroms. ${ }^{9}$ At room temperature and in $77 \mathrm{~K}$ solid solution, these features are very broad, $\sim 50-100$ nanometers. The narrowness of these lines suggest the vibrational energy of the fullerenes in the beam to be very low. These features are interpreted to result from coupling between several nearly degenerate forbidden singlet states. Increased vibrational energy will increase the coupling between these states and the ground state shortening the lifetime and broadening these features.

As further evidence of the ability of this design to produce beams of materials at cryogenic temperatures, figure 8 shows the formation of $\mathrm{C}_{60} \cdot \mathrm{Ar}$ and $\mathrm{C}_{70} \cdot \mathrm{Ar}$ van der Waals complexes. The fullerenes in this case were laser desorbed from a sublimed film on a copper surface. As many as twenty argon atoms have attached to $\mathrm{C}_{60}$. This data suggests significant vibration cooling of the fullerenes in the supersonic expansion.

An additional key piece of evidence that the delayed pressurization leads to better mixing and cooling resulted from photoelectron spectroscopy studies of $\mathrm{K}_{\mathrm{x}} \mathrm{C}_{60}$ using this source design for laser desorption. ${ }^{10}$ In these experiments, hotter clusters were formed when the delay was increased as indicated by the spectrum obtained. 


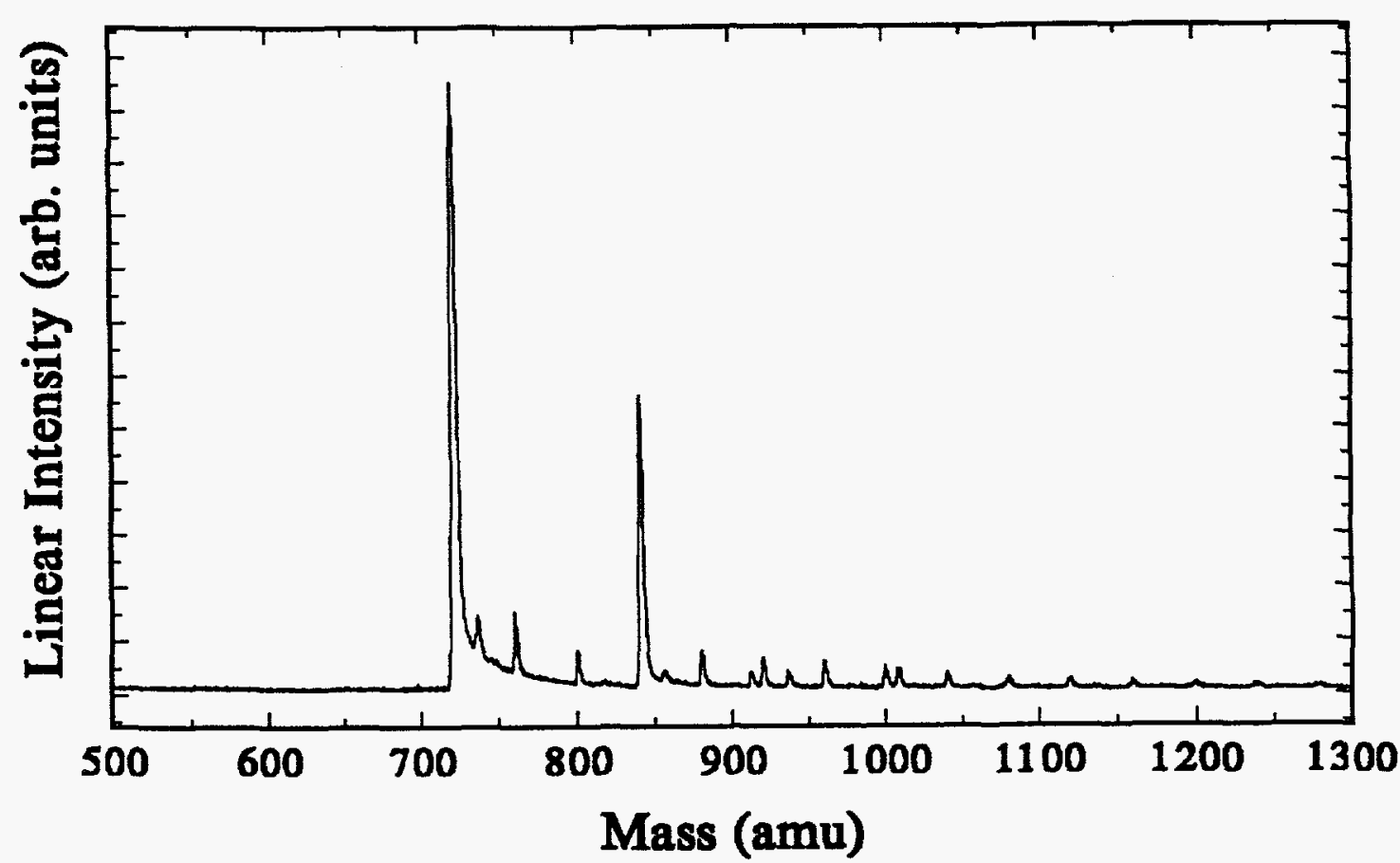

Figure 8. Reflectron time-of-flight mass spectrum of 532 nanometer laser desorbed $\mathrm{C}_{60}$ and $\mathrm{C}_{70}$ fullerenes with $1 \%$ argon in helium carrier gas. $\mathrm{C}_{60} \cdot \mathrm{Ar}_{\mathrm{x}}$ and $\mathrm{C}_{70} \cdot \mathrm{Ar}_{\mathrm{y}}$ complexes are observed. These neutral species were ionized using 157 excimer laser radiation.

\section{ACKNOWLEDGMENTS}

The authors wish to thank Professor R. E. Smalley for his contributions to this work. REH acknowledges support by an appointment to the U. S. Department of Energy Laboratory Cooperative Postgraduate Research Training Program administered by the Oak Ridge Institute for Science and Education. The publication was based upon research sponsored by Directors R\&D Fund and Office of Health and Environmental Research, Oak Ridge National Laboratory, U. S. Department of Energy, under contract DE-AC05-84OR21400 with Martin Marietta Energy Systems, Inc.

${ }^{1}$ T. G. Deitz, M. A. Duncan, D. E. Powers, and R. E. Smalley, J. Chem. Phys. 74, 6511 (1981).

${ }^{2}$ D. B. Geohegan, R. E. Haufler, R. L. Hettich, A. A. Puretzky, X. Zheng, and R. N. Compton, to appear in Proceedings of the 2nd Conference an Laser Ablation (1993).

${ }^{3}$ J. B. Anderson and J. B. Fenn, Phys. Fluids 8, 780 (1965).

${ }^{4}$ R. V. Ambartzumian, L. M. Dorozhkin, G. N. Makarov, A. A. Puretzky, and B. A. Chayanov, Appl. Phys. 22, 409 (1980).

${ }^{5}$ R. M. Jordan Company, Grass Valley, California.

${ }^{6}$ G. I. Dimov, Pribory i tecknica eksperimenta, 5, 168 (1968).

${ }^{7}$ S. C. O'Brien, J. R. Heath, R. F. Curl, and R. E. Smalley, J. Chem. Phys. 88, 220 (1988).

${ }^{8}$ T. Guo, M. D. Diener, Y. Chai, M. J. Alford, R. E. Haufler, S. M. McClure, T. Ohno, J. H. Weaver, G. E. Scuseria, and R. E. Smalley, Science 257, 1661 (1992).

${ }^{9}$ R. E. Haufler, Y. Chai, L. P. F. Chibante, M. R. Fraelich, R. B. Weisman, R. F. Curl, and R. E. Smalley, J. Chem. Phys. 95, 2197 (1991).

${ }^{10}$ L-S Wang, O. Cheshnovsky, J. P. Carpenter, S. J. Hwu, and R. E. Smalley, J. Chem. Phys. 96, 4028 (1992). 